\title{
Criminologie
}

\section{Le juge et la justice}

\section{Bernard Grenier}

Volume 15, numéro 1, 1982

Droit et justice

URI : https://id.erudit.org/iderudit/017153ar

DOI : https://doi.org/10.7202/017153ar

Aller au sommaire du numéro

Éditeur(s)

Les Presses de l'Université de Montréal

ISSN

0316-0041 (imprimé)

1492-1367 (numérique)

Découvrir la revue

Citer ce document

Grenier, B. (1982). Le juge et la justice. Criminologie, 15(1), 113-116.

https://doi.org/10.7202/017153ar

Ce document est protégé par la loi sur le droit d'auteur. L'utilisation des services d'Érudit (y compris la reproduction) est assujettie à sa politique d'utilisation que vous pouvez consulter en ligne.

https://apropos.erudit.org/fr/usagers/politique-dutilisation/
Cet article est diffusé et préservé par Érudit.

Érudit est un consortium interuniversitaire sans but lucratif composé de l’Université de Montréal, l'Université Laval et l'Université du Québec à Montréal. Il a pour mission la promotion et la valorisation de la recherche. https://www.erudit.org/fr/ 
Définir le rôle du juge sous l'éclairage de la rubrique "droit et justice " peut être d'une simplicité enfantine, voire d'une platitude désarmante. Ce rôle consiste à rendre la justice conformément à la règle de droit. Je pourrais ouvrir et clore cette réflexion par ce simple aphorisme qui résume en quelques mots la fonction du juge dans notre système juridique. Ce serait suffisant au plan théorique, mais tellement désincarné qu'il me semble opportun d'ajouter un peu de chair autour de la carcasse. Je le ferai à partir d'une expérience d'un an et demi à la Cour des sessions de la paix de Montréal. Il est bien entendu qu'il s'agit là d'une réflexion personnelle, toute empreinte de subjectivisme. Je ne prétends pas parler au nom des juges en général, ni même des collègues qui cuvrent au même tribunal que moi.

Le juge rend la justice conformément à la règle de droit : ces deux notions se compénètrent intimement. Leur interpénétration comporte un certain nombre de conséquences dignes de mention.

Parmi les règles juridiques qui encadrent le travail du juge, celles qui se rapportent à la preuve comptent parmi les plus importantes, dans la mesure où elles précisent les éléments qui sont admissibles et ceux qui ne le sont pas. C'est ainsi que mis à part certaines exceptions, la preuve par ouï-dire est irrecevable. Un témoin ne peut relater ce qu'une autre personne lui a dit; l'auteur de cette affirmation devra rapporter lui-même devant le tribunal les paroles qu'il a prononcées. Une autre règle de preuve interdit au témoin ordinaire d'émettre des hypothèses ou de tirer des conclusions; il ne dépose que sur les faits, et doit laisser au tribunal le soin de faire les déductions qui s'imposent. Une autre règle encore permet d'écarter de la preuve une déclaration faite à un policier, lorsque cet aveu est le résultat d'une promesse ou d'une menace émanant de lui. Il arrive que le juge refuse d'admettre en preuve un aveu qui correspond pourtant à la réalité, en raison du fait qu'il doute de son caractère libre et volontaire. Une dernière règle, probablement la plus fondamentale, exige de la couronne qu'elle prouve la culpabilité de l'accusé au-delà du doute raisonnable. Si le juge a donc "un doute raisonnable ", il doit en faire bénéficier l'accusé et l'acquitter. Il ne

* Juge, Sessions de la Paix, Montréal. 
peut condamner un individu à partir de simples soupçons.

Comme on peut le constater, l'existence des règles de la preuve, dont le paragraphe précédent ne cite que quelques exemples, impose au juge un cadre rigoureux auquel il doit se plier. Il est des éléments de preuve qu'on accepterait dans la vie de tous les jours, mais que le droit exclut pour des considérations de politique criminelle. Ce n'est pas parce qu'un journal annonce à grands renforts de publicité qu'un individu soupçonné d'un crime a été arrêté et est passé aux aveux, que cette personne sera nécessairement trouvée coupable. Ce n'est pas parce que les circonstances de la perpétration d'une infraction font planer certains soupçons au sujet d'un individu, que la preuve de sa culpabilité est établie au-delà du doute raisonnable. Un témoin à charge, par exemple un complice, peut rendre un témoignage accablant contre l'accusé. Mais si le contre-interrogatoire et l'ensemble de la preuve portent sérieusement atteinte à sa crédibilité, le juge considérera peut-être dangereux de condamner l'inculpé sur la seule foi de cette déposition, surtout si celle-ci n'est aucunement confirmée par une preuve indépendante reliant l'accusé au crime.

Dans notre système juridique dit accusatoire ou contradictoire, le juge n'a donc pas toute discrétion pour choisir ce dont il peut tenir compte pour rendre sa décision. Il doit se plier aux exigences des règles de preuve, de procédure et de droit de fond. La présence de ces contraintes juridiques fait en sorte que le procès n'a pas pour objectif premier la recherche de la vérité, mais bien la détermination de la culpabilité ou de l'innocence de l'accusé à l'intérieur des limites de la règle de droit. Si le procès visait uniquement à découvrir la vérité, tous les moyens pour y parvenir seraient acceptables et toutes les preuves seraient admissibles, quitte à ce que le juge en soupèse la valeur probante. La philosophie sous-jacente à notre droit oriente plutôt le procès vers la réponse à la question suivante : la preuve démontre-t-elle que l'accusé est coupable de l'accusation portée contre lui ?

L'intervention du juge se situe aussi à un autre niveau, ou mieux à une autre étape de la procédure, celle de la sentence. Si l'accusé est acquitté, tout est terminé et il n'est pas question d'imposer une peine à celui qui est jugé innocent de l'infraction reprochée. En revanche, s'il est déclaré coupable, le juge doit traduire au moyen d'une peine la réaction sociale au crime commis.

C'est un truisme de dire que la détermination de la peine constitue l'étape la plus difficile et la plus cruciale du processus pé- 
nal. On constate d'ailleurs, en conversant avec les accusés, que c'est leur grand sujet de préoccupation : quelle sentence vais-je avoir? Elle est cruciale car elle peut avoir des répercussions sur l'ensemble de la vie d'un individu; celui-ci risque de se voir priver de l'un de ses biens les plus chers, sa liberté.

C'est un moment difficile pour le juge qui a une décision importante à rendre mais qui dispose de très peu de balises pour le guider. La loi lui confère un très vaste pouvoir discrétionnaire dans la détermination de la peine. Elle se contente de préciser la peine maximale en rapport avec chaque infraction, et dans de rares cas la peine minimale. Certains crimes comme le vol qualifié, l'effraction dans une maison d'habitation, le viol ou la tentative de meurtre, comportent comme peine maximale l'emprisonnement à perpétuité. La sentence peut donc aller du sursis de sentence jusqu'à la prison à vie. Devant cet énorme éventail de possibilités, le juge ne dispose en définitive que de son bon sens et des grands principes énoncés par la jurisprudence pour imposer une peine. Il doit appliquer ces principes à la situation de l'individu qui est devant lui, en vertu de l'infraction dont il a été ou dont il s'est reconnu coupable.

Le choix de la sentence constitue sans aucun doute une décision angoissante, tant les conséquences sont considérables et les règles peu nombreuses. Il faut d'abord décider s'il convient d'imposer ou de ne pas imposer la prison. S'il est nécessaire de rendre une peine d'emprisonnement, il faut savoir si celle-ci sera de courte, moyenne ou longue durée. Une fois arrêté le type de durée, la décision la plus ardue est encore à venir : combien de jours, de semaines, de mois ou d'années? Il n'y a pas de magie dans les chiffres, il n'y a pas non plus de vérité absolue. Devant une même situation, plusieurs juges peuvent opter en faveur d'un quantum différent, compte tenu de leur perception des faits, de leur personnalité et de leur attitude face à certaines formes de déviance. En d'autres termes, et pour employer le mot fatidique, les sentences risquent d'être disparates.

Est-il inacceptable qu'il existe des variantes d'un juge à l'autre? Devrait-on, pour obvier à cette situation, adopter un tarif fixe pour chaque type d'infraction? Le débat dure depuis fort longtemps, et il ne m'appartient pas de le trancher. Qu'il me suffise d'exprimer l'avis qu'une tarification rigide des peines ne constitue pas la solution idéale. Une certaine souplesse est essentielle pour adapter la sentence à toutes les circonstances de l'affaire. L'adoption de principes directeurs susceptibles de guider le juge est plus que sou- 
haitable, mais il me semble essentiel de lui laisser une certaine marge de discrétion pour individualiser la peine.

Un dernier mot sur la façon de rendre la justice. Le stress est grand devant le tribunal, tant pour les témoins que pour l'accusé et, à un moindre degré, pour les avocats. Chacun a quelque chose à dire et veut à tout prix faire passer son message. Dans cette optique, le rôle du juge consiste à écouter d'une oreille attentive et à manifester de l'intérêt envers ceux qui s'adressent à lui. Ce n'est pas toujours facile et cela prend parfois du temps, mais c'est la rançon du métier de juge. Il se doit de laisser s'exprimer ceux qui sont devant lui sans les brusquer, sauf s'ils se répètent à outrance. J'ai compris du haut du banc le sens de l'expression anglaise : " to have his day in court ". Le témoin ou l'accusé ne viendra peut-être à la cour qu'une seule fois, et il veut avoir l'impression que ce jour lui est consacré. Voilà pourquoi la qualité de la justice est fonction non seulement de la valeur du jugement rendu, mais aussi de l'attitude d'accueil et de patience de celui qui préside le débat. Ce n'est pas toujours aisé, et pourtant c'est le principal objectif à atteindre pour que le justiciable ait l'impression qu'il a eu justice. 